\title{
Estudios de género y migración: Una revisión desde la perspectiva del siglo $\mathrm{XXI}_{[1]}$
}

\author{
Gender and Migration Scholarship: An Overview from a 21st Century \\ Perspective
}

Pierrette Hondagneu-Sotelo

University of Southern California, Estados Unidos sotelo@usc.edu

\begin{abstract}
Resumen
En los últimos treinta años la investigación sobre género y migración ha crecido de manera significativa, pero es necesario preguntarse dónde se encuentra hoy, a comienzos del siglo veintiuno. Gran parte de la investigación sobre inmigración muestra una ceguera androcéntrica continua hacia los asuntos feministas y de género (Morakvasic, 1984; Pedraza, 1991), situación que a pesar de no ser novedosa, sigue siendo real. Sin embargo, en este ensayo se presenta una historia diferente, ya que hoy sí existen estudios dinámicos sobre género y migración. La indagación académica sigue estando, de cierto modo, fragmentada y en este breve ensayo esbozo seis corrientes distintivas de investigación de género y migración.[2]
\end{abstract}

Pierrette Hondagneu-Sotelo,

Estudios de género y migración: Una revisión desde la perspectiva del siglo XXI Autoctonía. Revista de Ciencias Sociales e Historia, Vol. II, №1, Enero-Junio 2018, 26-36 ISSN 0719-8213

DOI: http://dx.doi.org/10.23854/autoc.v2i1.77 


\section{Género y migración: alzando la bandera}

En la primera categoría de los estudios de "género y migración" los investigadores, casi todos mujeres, prosiguen lo que algunos podrían llamar un enfoque dominante de las ciencias sociales. Aquí, el objetivo es hacer que el género sea una parte institucional de los estudios de inmigración. La finalidad no es, como a menudo se sugiere de forma errónea, únicamente medir los avances en materia de género para las mujeres inmigrantes y refugiadas. Por el contrario, un pequeño grupo de audaces académicos está enarbolando la bandera para establecer la legitimidad de los asuntos de género en los estudios de inmigración. En Estados Unidos esto incluye académicos prominentes como la socióloga, demógrafa y coeditora de la American Sociological Review, Katharine Donato, así como la historiadora y ex presidenta de la Social Science History Association Donna Gabbacia, y las antropólogas Patricia Pessar y Sarah Mahler.

Algunos de estos autores editaron un número especial de la International Migration Review (IMR) en 2006, con el título de "A Glass Half Full?: Gender in Migration Studies". Se trató de un seguimiento de veinte años a una edición especial de IMR de 1986, cuyo foco principal era la categoría de mujeres inmigrantes. Para la década de los 90s, la investigación había dejado de centrarse en las "mujeres" y hacía hincapié en la migración como un proceso de género. Este estudio buscó romper esquemas de género simplistas y llamó la atención sobre esta temática en los mercados laborales y redes sociales, el vínculo entre el trabajo remunerado y las relaciones familiares. Incluyó, además, los cambios en el patriarcado y la autoridad familiar, que se producen a través de la migración, y la vida transnacional marcada por el género y los grupos etarios (Pessar y Grasmuck, 1991, Kibria, 1993, Hondagneu-Sotelo, 1994). Más tarde, Stephanie Nawyn (2010) enfatizó las formas en que las ONG de reasentamiento de refugiados determinan la capacidad de estas mujeres para desafiar el patriarcado en el hogar, mientras reafirman el capitalismo patriarcal en el lugar de trabajo. El estudio de Cynthia Cranford (2007) enfatizó cómo la reestructuración económica, el trabajo y la política sindical permiten a las mujeres inmigrantes latinas, que se desempeñan como conserjes, desafiar las restricciones de género en múltiples ámbitos. En todos estos trabajos, se promovió el género como un elemento dinámico y constitutivo de la migración y la integración de los inmigrantes.

En el número especial de $I M R$, Donato et al. (2006) abordaron algunos de estos temas clave y ofrecieron una revisión multidisciplinaria del campo de la migración y de género. Los resultados de dicho trabajo reflejan el patrón identificado por Stacey y Thorne (1985) hace más de dos décadas: más apertura en la antropología, menos cambios en los campos más cuantitativos de la demografía y la economía. Investigadoras como Donato y Gabbaccia buscan descubrir lo que ellas llaman el "equilibrio de género" de los principales movimientos migratorios en todo el mundo y en diferentes períodos de tiempo. Ellas persiguen medir cuándo los flujos migratorios pasan de ser principalmente masculinos a ser en su gran mayoría femeninos, situación que ocurrió a nivel global en Estados Unidos a principios del siglo $\mathrm{XX}$.

En Europa, especialmente en España, hay una creciente nueva área de investigación sobre la maternidad transnacional y la migración laboral de mujeres sudamericanas a España y su papel como pioneras en la migración familiar (Escrivá, 2000, Pedone y Araujo, 2008). En Asia, la investigación se centra en los estudios de género, la migración y el estado (Oishi, 2005, Piper y Roces, 2003), mientras que en México, el país con la migración laboral transnacional en desarrollo continuo más prolongado, existe una variada investigación en el área de género (por ejemplo, Ariza, 2000, Arias, 2000, D’Aubeterre, 2000, Oehmichen, 
2000, Woo Morales, 1995, 2007). En Estados Unidos, un libro de Gordillo (2010) se centra en los vínculos transnacionales de género de las mujeres mexicanas, mientras que una publicación del año 2009 editado por Seyla Benhabib y Judith Resnick, lleva la bandera de género al territorio de los debates sobre ciudadanía, ley de inmigración, soberanía y jurisdicción legal. El tema de la violencia intrafamiliar en las vidas de las mujeres inmigrantes también ha obtenido justa atención (Menjívar y Salcido, 2002). Los anteriores son algunos de los diversos y continuos esfuerzos que buscan reformar los estudios de inmigración para que se reconozca la temática de género como fundamental para los procesos de migración.

\section{Migración y trabajo de cuidados}

Una segunda corriente de estudio se ha centrado exclusivamente en el vínculo entre la migración de las mujeres, el trabajo doméstico remunerado y la atención familiar. Los conceptos clave aquí son "trabajo de cuidados", "cadenas globales de cuidado", "déficit de cuidado", "maternidad transnacional" y "trabajo reproductivo social internacional". En este sentido, las teorías de la interseccionalidad han transformado profundamente el desarrollo de esta corriente de estudios. En Estados Unidos, a partir de la década de 1980, y guiados por el cambio de paradigma del trabajo de las académicas feministas de color, los conceptos unitarios de "hombres" y "mujeres" fueron reemplazados por la idea de que hay multiplicidades de feminidades y masculinidades, y que estos están interconectados, relacionados y entrelazados con las desigualdades de clase, raza, nacionalidad y sexualidad.

En esta recopilación de estudios de investigación, el enfoque se aleja de las relaciones entre mujeres y hombres acercándose a las desigualdades entre las mujeres inmigrantes y nación, junto con la forma en que estas se producen. Estas desigualdades se dan en el contexto internacional de alivio del trabajo reproductivo doméstico de las mujeres de los países ricos postindustriales, traspasándolo a las mujeres provenientes de países pobres de menor desarrollo, del Sur global. A menudo, esta situación exige separaciones familiares prolongadas entre las migrantes y sus hijos. Las investigaciones antes señaladas constituyen un gran conjunto de trabajos en constante crecimiento, sin embargo, es necesario mencionar los contribuyentes clave. Entre ellos se incluyen a Parrenas (2001), Chang (2000) y Hondagneu-Sotelo y Ávila (1997), Hondagneu-Sotelo $(2001,2007)$ en Estados Unidos; Constable (1997) y Lan (2006) en Asia; Lutz (2002, 2008), Escrivá (2000), Parrenas (2001) y Anderson (2000) en Europa y el Reino Unido; y el libro editado de Hochschild y Ehrenreich (2003), que cubre el terreno global. Las investigaciones más recientes examinan la integración de los hombres inmigrantes en trabajos domésticos, tales como los operarios polacos en Londres (Kilkey, 2010) y los jardineros inmigrantes mexicanos en Los Ángeles (Ramirez y HondagneuSotelo, 2009).

En este punto es necesario preguntarse el porqué esta literatura comenzó a surgir alrededor del año 2000. El pasado siglo XX estuvo marcado por el rápido aumento de las mujeres que migran para desempeñarse en el trabajo doméstico. Durante los períodos de mayor auge de la modernización y la industrialización, los inmigrantes eran principalmente hombres, por lo general provenientes de sociedades más pobres, a menudo coloniales, contratados para realizar "trabajos de hombres". Chinos, filipinos, japoneses, irlandeses, italianos y mexicanos, por ejemplo, fueron reclutados, cada uno a su vez, y llevados a Estados Unidos para construir infraestructura en el industrializado país. En algunos casos, se les permitió a los miembros de la familia unirse a estos hombres, pero en muchos otros, se les negó la admisión a mujeres y niños, en 
especial a aquellos que involucraban a grupos de inmigrantes percibidos como no blancos. Es más, la legislación gubernamental impuso estas prohibiciones a la incorporación permanente de estos trabajadores y sus familias. El Programa Bracero y el Programa de Trabajadores Invitados son ejemplos representativos de estos modernos sistemas enfocados en el género, que se basaron en el reclutamiento y subyugación del trabajo masculino y la exclusión de las familias.

Hoy en día las cosas han cambiado. Las fábricas se trasladan al extranjero en busca de mano de obra barata y los profesionales de alta tecnología y altamente educados se han unido a los trabajadores migrantes. Sin embargo, entre ellos hay legiones de mujeres que atraviesan el mundo, de sur a norte, de este a oeste para realizar trabajos domésticos remunerados. En consecuencia, en algunos lugares, es posible ver el cese de la mano de obra masculina migrante y la saturación de los mercados laborales para este mismo grupo de trabajadores. En lugares tan diversos como Italia, el Medio Oriente, Taiwán y Canadá, las mujeres migrantes filipinas que se desempeñan como niñeras y encargadas de la limpieza superan de manera amplia a los hombres provenientes del mismo país. En las distintas naciones, esta demanda se genera de modos diversos, lo que plantea dudas sobre cómo las políticas estatales facilitan la migración de las mujeres, y es aquí donde se presentan muchas variaciones. Lo que si está claro es que las mujeres de países tan diversos como Perú, Filipinas, Moldavia, Eritrea e Indonesia están abandonando a sus familias, comunidades y países para emigrar a miles de kilómetros de distancia y, así, desempeñarse en la nueva industria de crecimiento mundial que es la del trabajo doméstico remunerado y el de cuidado de ancianos. Sin embargo, lo que sorprende es la marginación de estos factores en los estudios sobre inmigración, circunstancia que podría explicarse por el hecho de que el tema reúne tres elementos que, por lo general, se consideran sin importancia como son las mujeres, la esfera doméstica y el trabajo de cuidado.

\section{Sexualidades}

Una tercera rama de la investigación de género e inmigración ha estado más relacionada con el área de las humanidades, los estudios queer y los estudios culturales. En este punto, la atención se centra en las sexualidades, incluidas las identidades homosexuales y queer, así como la heteronormatividad y la heterosexualidad obligatoria, empleadas en la inmigración legal tanto como formas de exclusión como de inclusión. El libro publicado de forma póstuma por Lionel Cantú, The Sexuality of Migration (2009), editado por su ex mentora Nancy Naples y su colega Salvador Ortiz, muestra cómo las relaciones sexuales entre hombres gays mexicanos están relacionadas con el turismo internacional, las redes transnacionales y, a veces, el asilo legal. Los debates sobre el matrimonio entre homosexuales también repercuten en las políticas migratorias que niegan la entrada a inmigrantes homosexuales, gays, lesbianas y transexuales. Eithne Luibheid (2002) retoma estos temas en Entry Denied: Controlling Sexuality at the Border, donde muestra cómo las definiciones implícitas y explícitas de heteronormatividad han sido fundamentales para las leyes que rigen el control de la inmigración. En la mayoría de las naciones, los ciudadanos heterosexuales pueden patrocinar a sus parejas de origen extranjero con el fin de obtener la residencia legal, pero solo 19 países de todo el mundo permiten que los ciudadanos gays y lesbianas lo hagan. Los Estados Unidos no forma parte dichas naciones. Como señalan los sociólogos Danielle Hidalgo y Carl Bankston (2010), la Ley de Inmigración norteamericana de 1965 convirtió al matrimonio heterosexual en la vía más importante para ingresar de forma legal a dicho país. Por lo general pensamos en esta Ley como una legislación de inmigración liberalizadora, ya que puso fin a las exclusiones raciales asiáticas y a la inmigración familiar legal institucionalizada. Sin embargo, esta normativa también es excluyente porque cosifica una 
definición heterosexual limitada de familia. Por otra parte, otro libro que aborda la permanente invisibilidad de los inmigrantes queer y homosexuales es Global Divas: Filipino Gay Men in the Diaspora de Martin Manalansan (2003), una etnografía realizada en la ciudad de Nueva York.

Con demasiada frecuencia, el concepto de las "sexualidades" se traduce en un enfoque homosexual (sexualidad queer), ámbito en el que Gloria González López, con su libro Erotic Journeys: Mexican Immigrant Women and Sex Lives (2005) realiza una valiosa contribución y una importante intervención en el estudio profundo sobre el tema. En él se analizan las prácticas normativas heterosexuales y los valores de las mujeres trabajadoras inmigrantes mexicanas, cuyo objetivo es revelar cómo los procesos invisibles de poder organizan las vidas de estas personas. En lugar de adoptar el enfoque familiar centrado en problemas sociales, como el embarazo adolescente o la transmisión a nivel transnacional del VIH, González López examina las prácticas sexuales de las mujeres inmigrantes mexicanas y cómo se sienten con respecto a ellas. Este trabajo representa la imaginación sociológica en su mejor momento, al hacer visible la construcción social y la naturaleza problemática de algo previamente considerado como normativo y aceptable.

\section{Tráfico sexual}

La cuarta corriente de investigación sobre género y migración se centra en los debates sobre el tráfico sexual y las mujeres migrantes involucradas en el comercio sexual. En Europa, esta es una gran área de investigación y activismo, en la que la cruzada moral suele ocultar las estructuras de explotación laboral (Anderson y Davidson, 2003, Anderson y Andrijasevic, 2008, Oso, 2010). Uno de los críticos más fuertes de la "industria del rescate" es la académica y activista Laura Agustín (2007). Originaria de América Latina, pero radicada en el Reino Unido, esta investigadora mantiene un blog muy actualizado en donde se ofrecen comentarios sobre el turismo sexual, la migración del trabajo sexual y las severas medidas de la policía y las autoridades de inmigración. El trabajo sexual atrae a mujeres inmigrantes de Europa del Este, el Caribe y América Latina, Asia y África. Con una alta influencia del pensamiento fronterizo de Anzaldúa, Agustín busca romper la dualidad con la que se ve a los migrantes, ya sea como intrusos no deseados o víctimas indefensas. Observa el trabajo sexual de las mujeres migrantes a través del lente de los mercados laborales y las economías informales, y favorece una perspectiva que carece de moralización, una en la que prima la agencia por sobre la victimización.

La académica y especialista en temáticas relacionadas con Estados Unidos, Rhacel Parrenas, más conocida por su labor con las trabajadoras domésticas filipinas trasnacionales y sus estructuras familiares, se encuentra escribiendo sobre su investigación acerca de los entertainers[3] migrantes filipinos y hostesses[4] en Japón. Parte de este informe ya apareció como un capítulo en su libro del año 2008, The Force of Domesticity.

Al igual que Laura Agustín, Parrenas ve a las trabajadoras sexuales migrantes desde la postura de los mercados laborales y las limitaciones estructurales, en lugar de considerarlas como mujeres inmorales o desafortunadas víctimas de la explotación. A diferencia de Agustín, Parrenas ofrece una etnografía detallada de la dependencia que tienen las industrias sexuales japonesas de mujeres filipinas, hostesses y entertainers transgéneros. Hasta hace muy poco, existía un sistema completo de visas para facilitar contratos de trabajo temporales a hostesses filipinas en Japón, pero se le dio término debido a la presión de Estados Unidos a 
partir de la "guerra contra el tráfico" que supone que todas las transacciones sexuales comerciales equivalen a explotación, independiente del consentimiento. Los Estados Unidos financian más de 100 proyectos en todo el mundo para detener el tráfico sexual. Parrenas y Agustín están de acuerdo con la postura de que muchas de estas campañas estadounidenses corresponden a herramientas para controlar a las mujeres y para difundir la cultura y la moral colonialista de dicho país.

\section{Fronteras y migración}

El quinto es un ámbito de estudio amplio y debe su legado al clásico de Gloria Anzaldúa, Borderlands/ La Frontera: The New Mestiza, publicado en 1987. La investigación que este libro generó reúne un enfoque de estudios chicanos sobre el carácter hibrido de las identidades y del espacio en las fronteras. Influenciado por el pensamiento feminista socialista y el colonialismo interno, el enfoque se centra tanto en la identidad mestiza, como en los espacios que desafían la fácil oposición existente entre dominante y dominado a ambos lados de la frontera. Women and Migration in the US-Mexico Borderlands, editado por Denise Segura y Pat Zavella (2007), es el mejor ejemplo de esta corriente. En él, los participantes argumentan que hay fronteras feministas y el énfasis teórico posee características de índole estructural, discursivo, interaccional y agente. Los nuevos objetivos de investigación que se centran en la recepción de género para los inmigrantes mexicanos, tanto en el sur como en el medio oeste, también hacen hincapié en las fronteras y los cruces diversos (Deeb-Sossa y Binkham Mendez 2008, Schmalzbauer, 2009).

La noción de una "zona fronteriza de género" repercute en la investigación mucho más allá del límite entre los Estados Unidos y México. Como ya se mencionó, Laura Agustín, la académica y activista que se enfoca en el tráfico sexual, también está inspirada por Anzaldúa y de forma deliberada utiliza el pensamiento fronterizo, desafiando la presunta opresión y victimización de las trabajadoras sexuales migrantes, y reconsiderando los derechos migratorios de las mujeres en un contexto más amplio. La investigación de Bandana Purkayastha (2003) sobre las mujeres inmigrantes del sur de Asia también reúne las interseccionalidades y la vida social transnacional. Yen Le Espíritu (2003) centra la atención en Estados Unidos como el principal "cruzafronteras", al destacar en su trabajo el papel del imperialismo estadounidense, la intervención militar y las corporaciones multinacionales en el fomento de los movimientos de refugiados y la migración laboral y profesional. Lo anterior constituye un giro académico en el antiguo eslogan presente en las camisetas chicanas: "No cruzamos la frontera. Esta nos cruzó" lo que, de igual modo, resuena en el lema político utilizado por los activistas inmigrantes del Caribe y el sur de Asia en el Reino Unido, que señala el legado colonialista de la migración contemporánea y las transiciones demográficas: "Estamos aquí porque estuviste allí".

\section{Género, migración y niños}

Un área emergente de estudio se centra en el género, la migración y los niños. Con un desarrollo menos cohesivo que los otros ámbitos ya examinados, la investigación sobre los niños y las repercuciones de género de la migración transnacional es, sin embargo, un campo emergente crucial. Las construcciones sociales de género de la niñez median los procesos migratorios transnacionales y las infancias (Orellana, et al., 2003; Thorne, et al., 2003). Los investigadores han examinado las dinámicas de género que rodean a "los niños dejados atrás" cuando sus madres emigran como trabajadoras domésticas transnacionales (Parrenas, 2005); los relatos consensuados sobre sexualidad y pureza entre mujeres jóvenes de segunda generación 
(Espiritu, 2001); las experiencias laborales de género y raciales de la juventud de segunda generación (López, 2003); y las preocupaciones y estrategias de género que emplean los padres inmigrantes al organizar los viajes transnacionales de sus hijos a sus hogares (Smith, 2005). Otro corpus de estudio considera el trabajo de género realizado por los hijos de inmigrantes mexicanos pobres y de clase trabajadora (Valenzuela, 1999; Estrada y Hondagneu-Sotelo, 2011). La investigación también ha examinado las expectativas de género de los niños con respecto a los proyectos de migración familiar (Pavez Soto, 2010) y, en términos más generales, las opciones de género para continuar estudios versus la migración (París, 2010).

\section{Conclusión}

Como se ha tratado de demostrar en el presente ensayo, el impulso en los estudios de género y migración avanza en muchas direcciones. En ellos se incluyen nuevas y constantes investigaciones sobre las cadenas globales de cuidado, procesos del mercado laboral y campañas de activismo en torno al trabajo sexual y en contra del tráfico ligado a éste. Mujeres e hibridez fronteriza, proyectos permanentes sobre los procesos de migración transnacional marcados por el género y niveles generacionales, construcciones sociales de género de la niñez y avanzadas tabulaciones en demografía son otras áreas de investigación. Muchos de estos implican un cambio sutil desde un paradigma focalizado en "migración y desarrollo" hacia uno que se centra en el género y la "integración de inmigrantes". A pesar de que creo que todos estos estudios son valiosos, existen dos tendencias destacables. En primer lugar, en su mayoría, los investigadores en estas diferentes áreas no se comunican entre sí. En segundo lugar, existe una sordera casi total de los académicos que trabajan en otros ámbitos principales de los estudios de inmigración, los que incluyen la asimilación segmentada, la religión de los inmigrantes, el transnacionalismo y la ciudadanía. Lo primero se debe a la naturaleza cada vez más especializada y fragmentada de la investigación en ciencias sociales hoy en día, y lo último persiste como un problema que debe remediarse.

\section{Bibliografía}

Agustín, Laura María, 2007, Sex at the Margins: Migration, Labour Markets and the Rescue Industry, London/New York, Zed Books.

Anderson, Bridgit, 2000, Doing the Dirty Work: The Global Politics of Domestic Labour, London/ New York, Zed Books.

Anderson, Bridgit and Julia O'Connell Davidson, 2003, Is Trafficking in Human Beings Demand Driven?: A Multicountry Pilot Study, Geneva, International Organization for Migration.

Anderson, Bridgit, and Rutvica Andrijasevic, 2008, "Sex, Slaves and Citizens: The Politics of AntiTrafficking", Soundings, num. 40, pp. 135145.

Arias, Patricia, 2000, "Las migrantes de ayer y de hoy", en Dalia Barrera Bassols, and Cristina Oehmichen Bazán (eds.), Migración y relaciones de género en México, México, GIMTRAP/ UNAM, IIA, pp. 185202.

Ariza, Marina, 2000, "Género y migración femenina: Dimensiones analíticas y desafíos metodológicos", en Dalia Barrera Bassols, and Cristina Oehmichen Bazán (eds.), Migración y relaciones de género en México, México, GIMTRAP/UNAM, IIA, pp. 3362. 
Benhabib, Seyla and Judith Resnick (eds.), 2009, Migrations and Mobilities: Citizenship, Borders and Gender, New York, New York University Press.

Cantú, Lionel, 2009, The Sexuality of Migration: Border Crossings and Mexican Immigrant Men, edited by Nancy A. Naples, and Salvador VidalOrtiz, New York/London, New York University Press.

Chang, Grace, 2000, Disposable Domestics: Immigrant Women Workers in the Global Economy, Cambridge, Massachusetts, South End Press.

Constable, Nicole, 1997, Maid to Order in Hong Kong: Stories of Filipina Workers, Ithaca, Cornell University Press.

Cranford, Cynthia J., 2007, "It's Time to Leave Machismo Behind!: Challenging Gender Inequality in an Immigrant Union", Gender \& Society, vol. 21, num. 3, pp. 409436.

D’Aubeterre Buznego, María Eugenia, 2000, El pago de la novia. Matrimonio, vida conyugal y prácticas transnacionales en San Miguel Acuexcomac, Puebla, Zamora, El Colegio de Michoacán/Benemérita Universidad Autónoma de Puebla, Instituto de Ciencias Sociales y Humanidades.

DeebSossa, Natalia and Jennifer Binkham Mendez, 2008, "Enforcing Borders in the Nuevo South: Gender and Migration in Williamsburg, Virginia and the Research Triangle, North Carolina", Gender \& Society, vol. 22, num. 5, pp. 613638.
Donato, Katharine M. et al., 2006, “A Glass Half Full? Gender in Migration Studies", International Migration Review, vol. 40, num. 1, pp. 326.

Escrivá, Ángeles, 2000, “Empleadas de por vida? Peruanas en el servicio doméstico en Barcelona", Papers, num. 60, pp. 327-342.

Espiritu, Yen Len, 2001, "We don't Sleep Around Like White Girls Do': Family, Culture, and Gender in Filipina American Lives", Signs, vol. 26, num. 2, pp. 415440.

Espiritu, Yen Len, 2003, Home Bound: Filipino American Lives Across Cultures, Communities, and Countries, Berkeley/Los Angeles, University of California Press.

Estrada, Emir and Pierrette HondagneuSotelo, 2011, "Intersectional Dignities: Latina Immigrant Adolescent Street Vendors in Los Angeles", Journal of Contemporary Ethnography, vol. 40, num. 1.

GonzálezLópez, Gloria, 2005, Erotic Journeys: Mexican Immigrant Women and their Sex Lives, Berkeley/Los Angeles, University of California Press.

Gordillo, Luz María, 2010, Mexican Women and the Other Side of Immigration: Engendering Transnational Ties, Austin, University of Texas Press.

Grasmuck, Sharri and Patricia R. Pessar, 1991, Between Two Islands: Dominican International Migration, Berkeley/Los Angeles, University of California Press.

Hidalgo, Danielle Antoinette and Carl Bankston III, 2010, "Reinforcing Polarizations: U.S. Immigration and the Prospect of Gay Marriage", 
Sociological Spectrum, vol. 30, num. 1, pp. 429.

Hochschild, Arlie and Barbara Ehrenreich (eds.), 2003, Global Woman: Nannies, Maids and Sex Workers in the New Economy, New York, Metropolitan Books.

HondagneuSotelo, Pierrette, 1994, Gendered Transitions: Mexican Experiences of Immigration, 4th printing, Berkeley/Los Angeles, University of California Press.

HondagneuSotelo, Pierrette, 2001, Domestica: Immigrant Workers Cleaning and Caring in the Shadows of Affluence, Berkeley/ Los Angeles, University of California Press.

HondagneuSotelo, Pierrette, 2007, “The Domestic Goes Global" (new preface), Domestica: Immigrant Workers Cleaning and Caring in the Shadows of Affluence, 2nd. ed., Berkeley/Los Angeles, University of California Press (Spanish language translation, Mexico, Miguel Ángel Porrúa, forthcoming 2011).

HondagneuSotelo, Pierrette and Ernestine Avila, 1997, "I'm Here, But I'm There: The Meanings of Latina Transnational Motherhood", Gender \& Society, vol. 11, num 5, pp. 548571.

Kibria, Nazli, 1993, Family Tightrope: The Changing Lives of Vietnamese Americans, Princeton, New Jersey, Princeton University Press.

Kilkey, Majella, 2010, "DomesticSector Work in the UK: Locating Men in the Configuration of Gendered Care and Migration Regimes", Social Policy and Society, num. 9, pp. 443454.
Lan, PeiChia, 2006, Global Cinderellas: Migrant Domestics and Newly Rich Employers in Taiwan, Duke University Press.

Lopez, Nancy, 2003, Hopeful Girls, Troubled Boys: Race and Gender Disparity in Urban Education, New York, Routledge.

Lubheid,Eithne, 2002, Entry Denied: Controlling Sexuality at the Border, Minneapolis, University of Minnesota Press.

Lutz, Helma, 2002, "At Your Service Madam! The Globalization of Domestic Service", Feminist Review, num. 70, pp. 89104.

Lutz, Helma (ed.), 2008, Migration and Domestic Work: A European Perspective on a Global Theme, London, Ashgate Press.

Manalansan, Martin, 2003, Global Divas: Filipino Gay Men in the Diaspora, Durham, North Carolina, Duke University Press.

Menjívar, Cecilia and Olivia Salcido, 2002, "Immigrant Women and Domestic Violence: Common Experiences in Different Countries", Gender and Society, vol. 16, num. 6, pp. 898920.

Morakvasic, Mirjana, 1984, "Birds of Passage are also Women...", International Migration Review, vol. 18, num. 4, pp. 886907.

Nawyn, Stephanie J., 2010, "Institutional Structures of Opportunity in Refugee Resettlement: Gender, Race/Ethnicity, and Refugee ngos", Journal of Sociology \& Social Welfare, vol. 37, num. 1, March, pp. 149167. 
Oehmichen, Cristina, 2000, "Las mujeres indígenas migrantes en la comunidad extraterritorial", in en Dalia Barrera Bassols, and Cristina Oehmichen Bazán (eds,), Migración y relaciones de género en México, México, GIMTRAP/UNAM, IIA, pp. 319349.

Oishi, Nana, 2005, Women in Motion: Globalization, State Polices, and Labor Migration in Asia, Stanford, Stanford University Press.

Oso Casas, Laura, 2010, “Money, Sex, Love and the Family: Economic and Affective Strategies of Latin American Sex Workers in Spain”, Journal of Ethnic and Migration Studies, vol. 36, num. 1, pp. 4765.

Orellana, Marjorie Faulstich et al., 2003, "Transnational Childhoods: The Participation of Children in Processes of Family Migration", Social Problems, vol. 48, num. 4, pp. 572591.

París Pombo, María Dolores, 2010, "Youth Identities and the Migratory Culture among Triqui and Mixtec Boys and Girls", Migraciones Internacionales, vol. 5, num. 4, pp. 139164.

Parrenas, Rhacel,2001, Servants of Globalization, Stanford, Stanford University Press.

Parrenas, Rhacel, 2005, Children of Global Migration: Transnational Migration and Gendered Woes, Stanford, Stanford University Press.

Parrenas, Rhacel, 2008, The Force of Domesticity: Filipina Migrants and Globalization, New York, New York University Press.

Pavez Soto, Izkra, 2010, "Peruvian Girls and
Boys as Actors of Family Migration in Barcelona: Generational Relations and Expectations", Migraciones Internacionales, 19, vol. 5, num. 4, pp. 6999.

Pedone, Claudia and Sandra Gil Araujo, 2008, "Maternidades transnacionales entre América Latina y el Estado español: El impacto de las políticas migratorias en las estrategias de reagrupación familiar", in Carlota Sole, Sonia Parella, and Leonardo Cavalcanti (coords.), Nuevos retos del transnacionalismo en el estudio de las migraciones, Madrid, Observatorio Permanente de la Inmigración.

Pedraza, Silvia, 1991, "Women and Migration: The Social Consequences of Gender", Annual Review of Sociology, num. 17, pp. 303325.

Piper, Nicola and Mina Roces (eds.), 2003, Wife or Worker? Asian Women and Migration, Oxford, Rowman and Littlefield Publishers.

Purkayastha, Bandana, 2003, "Skilled Migration and Cumulative Disadvantage: The Case of Highly Qualified Asian Indian Immigrant Women in the U.S.”, Geoforum, vol. 36, num. 2, pp. 181196.

Ramírez, Hernán and Pierrette HondagneuSotelo, 2009, "Mexican Immigrant Gardeners in Los Angeles: Entrepreneurs or Exploited Workers?", Social Problems, vol. 56, num. 1, pp. 7088 .

chmalzbauer, Leah, 2009, "Gender on a New Frontier: Mexican Migration in the Rural Mountain West", Gender \& Society, vol. 23, num. 6, pp. 747767. 
Segura, Denise and Patricia Zavella, 2007, Women and Migration in the U.S.-Mexico Borderlands, North Carolina, Duke University Press.

Smith, Robert Courtney, 2005, Mexican New York: Transnational Lives of New Immigrants, Berkeley/Los Angeles, University of California Press.

Stacey, Judith and Barrie Thorne, 1985, "The Missing Feminist Revolution in Sociology", Social Problems, vol. 32, num. 4, pp. 301316.

Thorne, Barrie et al., 2003, "Raising Children, and Growing Up, Across National Borders: Comparative Perspectives on Age, Gender, and Migration", in Pierrette HondagneuSotelo (ed.), Gender and U.S. Immigration: Contemporary Trends, Berkeley/ Los Angeles, University of California Press, pp. 241262.

Valenzuela, Abel, 1999, "Gender Roles and Settlement Activities Among Children and Their Immigrant Families", American Behavioral Scientist, vol. 42, num. 4, pp. 720742.

Woo Morales, Ofelia, 1995, "Las mujeres mexicanas indocumentadas en la migración internacional y la movilidad transfronteriza", in Soledad González, et al. (eds.), Mujeres, migración y maquila en la frontera norte, México, D. F., El Colegio de la Frontera Norte/El Colegio de México, pp. 6587.

Woo Morales, Ofelia, 2007, "La experiencia migratoria de las mujeres urbanas hacia el norte", in Patricia Arias, and Ofelia Woo Morales (coords.), ¿Campo o ciudad? Nuevos espacios y formas de vida, Guadalajara, Universidad de Guadalajara.
Recibido: 05 de septiembre de 2017

Aceptado: 04 de enero 2018

NOTAS

[1] Artículo publicado originalmente en: Hondagneu-Sotelo, Pierrette. (2011). "Gender and Migration Scholarship: An Overview from a 21st Century Perspective". Revista Migraciones Internacionales, Vol. $6 \mathrm{~N}^{\mathrm{o}} 1, \mathrm{pp}$. 219-233. Publicado aquí con autorización de la autora, Pierrette Hondagneu-Sotelo y del Editor, Eduardo Rodríguez, de la Revista Migraciones Internacionales. Traducido por Daniela Soto y Dr. Juan Eduardo Ortiz López. Traducción revisada por Dra. Carol Chan y Dra. Iskra Pavez-Soto. [Nota del Editor].

[2] En consideración a las limitaciones de espacio para este breve ensayo, la presente revisión no constituye una descripción completa de los estudios sobre género y migración, sino más bien un resumen analítico de las tendencias recientes en esta subárea. Nota traducida de la version original.

[3] El concepto entertainers puede traducirse al español como una persona que trabaja en el área de la entretención o del espectáculo; también alude a un artista o un performer [N. del T].

[4] El concepto hostesses puede traducirse al español como azafatas o anfitrionas; también hace referencia a personas que trabajan en servicios de acompañamiento, los cuales pueden ser de carácter sexual o no [N. del T]. 